\title{
A PARTICLE SYSTEM WITH EXPLOSIONS: LAW OF LARGE NUMBERS FOR THE DENSITY OF PARTICLES AND THE BLOW-UP TIME.
}

\author{
TERTULIANO FRANCO AND PABLO GROISMAN
}

\begin{abstract}
Consider a system of independent random walks in the discrete torus with creation-annihilation of particles and possible explosion of the total number of particles in finite time. Rescaling space and rates for diffusion/creation/annihilation of particles, we obtain a stong law of large numbers for the density of particles in the supremum norm. The limiting object is a classical solution to the semilinear heat equation $\partial_{t} u=\partial_{x x} u+f(u)$. If $f(u)=u^{p}, 1<p \leq 3$, we also obtain a law of large numbers for the explosion time.
\end{abstract}

\section{INTRODUCTION}

We consider nearest-neighbors symmetric independent random walks superposed with birth and death dynamics in the discrete torus. At rate one, each particle jumps to one of its neighbors with the same probability. In addition, if at a site there are $r$ particles, at that site a new particle is created at rate $b(r)$ and a particle is destroyed at rate $d(r)$.

We study suitable scaled versions of this process, that (as will be shown) converge almost surely in the $L^{\infty}$-norm to the solution of the semilinear parabolic problem

$$
\begin{cases}u_{t}=u_{x x}+f(u) & (x, t) \in \mathbb{T} \times[0, T) \\ u(x, 0)=\varphi(x) \geq 0 & x \in \mathbb{T}\end{cases}
$$

where $f=b-d$ is assumed to be smooth, $\varphi$ is smooth and nonnegative, and $\mathbb{T}$ denotes the continuous one-dimensional torus. This equation has been widely studied in the literature, being used to model diverse processes in mechanics, physics, chemistry, technology, biology and many other areas. For instance, under certain conditions, it describes conduction in plasma, gas filtration and liquids in porous media, chemical reactions, processes of growth and migration of populations, etc.

One of the most remarkable properties of this equation is the possible occurrence of singularities due to the presence of the nonlinear source $f$. Even starting from regular data, for which there exist an existence, uniqueness and continuous dependence theory for short times, the solution may develop singularities in finite time. Although for linear evolution problems singularities may occur, they appear due to singularities in the coefficients or in the problem data, while in this case, singularities appear because of the nonlinear essence of the equation, and the time and space localization of them has to be determined through a careful analysis.

In this problem, singularities appear in the simplest way: they are due to a fast increasing of the solution that leads the $L^{\infty}$ norm to grow-up to infinity in a finite time $T_{\max }=T_{\max }(\varphi)$.

The phenomenon is known as blow-up, and is interpreted as an abrupt change in the order of magnitude in the modeled quantity. It was successfully used to model, for instance, explosions in exothermic chemical reactions, population dynamics, fatigue cracking (in this

2010 Mathematics Subject Classification. 60K35, 35K55, 35B40.

Key words and phrases. Hydrodynamic limit, Parabolic equations, blow-up. 
case explosion means that a microscopic crack changes its scale and becomes macroscopic, indicating a crack in the material due to fatigue).

In view of this, it is important to understand the microscopic behavior of this kind of systems, and in particular their singularities (presence of them, space-time location, order, etc.).

A well known condition on the nonlinear term $f$ that assures the existence of solutions with blow-up is being convex, strictly positive in some interval $[a,+\infty)$ and

$$
\int_{a}^{\infty} \frac{d s}{f(s)}<\infty
$$

The most simple source verifying this conditions is $f(s)=s^{p}, p>1$. For a general description of the blow-up problem we refer the reader to the books $[14,15]$ and the surveys $[2,7]$.

Coming back to the Markov chain (the particle system), the scaling here is in the same spirit of $[3,4,9,10]$. The initial quantity of particles per site is also rescaled, different than in the hydrodynamic limit context [8].

As main results, we obtain almost sure convergence for the density of particles in the supremum norm for any compact time interval not containing $T_{\max }$.

This result was obtained by Blount, [4] for $f$ a polynomial with negative leading term. In that case solutions are known to be bounded for every $t \geq 0$ and hence globally defined, see also $[1,3,9,10]$.

The first part of the proof consists in proving the result for birth rates $b$ with compact support, where there is no blow-up and the solution is bounded. This follows essentially the work of Blount [4], the main difference being that we consider any continuously differentiable $f$ instead of polynomials. In the second part of the proof, we couple a sequence of Markov chains as the one described above, with birth rates $b_{j}$, where $\left(b_{j}\right)_{j \geq 1}$ is a sequence of smooth functions with compact support approximating $b$. This coupling allows us to extend the result proved in the first part to any smooth $b$, including those one satisfying (2).

As an immediate corollary, the liminf of the explosion times of the discrete systems is no smaller than the blow-up time of the solution to the PDE (1). The opposite inequality is much harder and we are only able to obtain it in some specific cases. Assuming that $f(s)$ verifies (2) and that $d$ is a bounded or linear function, we prove that, for each $N \geq 1$, the corresponding particle system explodes with probability one. Under the additional condition $f(s)=b(s)-d(s)=s^{p}$, with $1<p \leq 3$ we also prove that the explosion time of the particle system converges in probability, as $N \rightarrow \infty$, to the blow-up time of the solution to the PDE.

We remark here that if $f$ is globally Lipschitz then there is no explosion with probability one.

To the best of our knowledge, there is no previous work about the limit density of interacting particle systems exhibiting blow-up. For instance, $[3,4,9,10]$ in this type of scaling, and [11] in the hydrodynamical limit context, have considered creation of particles, in some cases with unbounded limit, but all of them deal with processes defined for all times.

It is worth to notice that since the particle system explodes in finite time, the expectation of the number of particles is infinity at any positive time. Hence, any method based on expectations is doomed to fail. This motivates the use of couplings. 


\section{Notation AND RESUlts}

2.1. The particle system. Denote $\mathbb{T}_{N}=\mathbb{Z} /(N \mathbb{Z})$ the discrete torus with $N$ points. Fix two nonnegative smooth functions $b, d: \mathbb{R}_{+} \rightarrow \mathbb{R}_{+}$such that $d(0)=0$. We consider also a parameter $\ell \in \mathbb{N}$, that represents the number of particles per site in the initial configuration (this is clarified in assumption (A1) of Theorem 2.1 below). We characterize the continuous time Markov chain

$$
(\eta(t))_{t \geq 0}=\left(\eta_{1}(t), \ldots, \eta_{N}(t)\right)_{t \geq 0}
$$

with state space $\Omega_{N}=\mathbb{N}^{\mathbb{T}_{N}} \cup\{\infty\}$ by its jump rates given by

- at rate $N^{2} \eta_{k}$, a particle jumps from $k$ to $k+1$;

- at rate $N^{2} \eta_{k}$, a particle jumps from $k$ to $k-1$;

- at rate $\ell b\left(\ell^{-1} \eta_{k}\right)$, a new particle is created at $k$;

- at rate $\ell d\left(\ell^{-1} \eta_{k}\right)$, a particle is destroyed at $k$.

The transitions above are assumed for all $k \in \mathbb{T}_{N}$. Aiming not carry on the notation, we do not index $\eta(t)$ on $N$ and on $\ell$. Since there are no assumptions on the behavior of $b$ at infinity, the waiting times of this Markov chain can be summable. If that is the case we say that the process explodes or blows up, and we define the state of the process as $\infty$ for times greater or equal than the sum of the waiting times, that we call $T_{\max }^{N}$. More precisely, define

$$
T_{M}^{N}:=\inf \left\{t \geq 0:\|\eta(t)\|_{\infty} \geq \ell M\right\} \text { and } T_{\max }^{N}:=\lim _{M \rightarrow \infty} T_{M}^{N} .
$$

Hence we can easily define $\eta(t)$ for $t<T_{\max }^{N}$ and we define $\eta(t)=\infty$ for $t \geq T_{\max }^{N}$. A graphical construction of this process is given in Section 3. For more on explosions of Markov chains we refer to [12].

Next, we define the spatial density of particles $X^{N}$, which is a function defined on the continuum torus $\mathbb{T}=[0,1]$, identifying 0 and 1 and considering a fixed orientation.

For $k \in \mathbb{T}_{N}$, let $x_{k}=k / N$ and define

$$
X^{N}\left(x_{k}, t\right)=\ell^{-1} \eta_{k}(t) .
$$

Also for $x_{k}<x<x_{k+1}$, we define the density of particles by linear interpolation, i.e.

$$
X^{N}(x, t)=(N x-k) X^{N}\left(x_{k+1}, t\right)+(k+1-N x) X^{N}\left(x_{k}, t\right) .
$$

If $\eta(t)=\infty$, we say that $\left\|X^{N}(\cdot, t)\right\|_{\infty}=\infty$ as well. We point out that this interpolation has no special meaning. Using instead a smoother interpolation or even defining $X^{N}$ as a step function would not change the results.

2.2. The partial differential equation. We make the following assumptions on the data of problem (1):

- The initial datum $\varphi$ is $C^{4}$ and nonnegative.

- The source term $f$ is $C^{1}$ and $f(0) \geq 0$.

Under the above assumptions this equation has a unique (local) solution $u$, which is smooth in some interval $\left(0, T_{\max }\right)$. Here $T_{\max }$ is the maximal existence time. If $f$ is globally Lipschitz then $T_{\max }=+\infty$ (global existence) but it can be proved that if $f$ is convex and verifies (2) then, for positive $\varphi$, the solution blows up in finite time, meaning that it is smooth in $\left(0, T_{\max }\right)$, but

$$
\lim _{t \nearrow T_{\max }}\|u(\cdot, t)\|_{\infty}=\infty
$$


These results are the content of Theorem 6.1 in Section 6. For general references on the study of this equation, existence, uniqueness and asymptotic behavior (including the blow-up case) see the books $[13,14,15,16]$.

The partial differential equation (1) satisfies a comparison principle. Since we require $f(0) \geq 0, u \equiv 0$ is a sub-solution to this problem and hence for nonnegative initial data, the solution is positive. All the regularity assumptions on the data of the problem are to guarantee the smoothness of the solution. They can be relaxed to some extent, but since we are not focused on the problems arising due to the lack of regularity of the solutions, we prefer not to include them to simplify the exposition.

We are in a position to state the main results of this paper.

Theorem 2.1. Assume that

(A1) $\left\|X^{N}(\cdot, 0)-\varphi(\cdot)\right\|_{\infty} \rightarrow 0$ almost surely;

(A2) for any $c>0, \ell=\ell(N)$ satisfies $\sum_{N \geq 0} N^{3} e^{-c \ell}<\infty$.

Then, for any $T \in\left[0, T_{\max }\right)$,

$$
\lim _{N \rightarrow \infty} \sup _{t \in[0, T]}\left\|X^{N}(\cdot, t)-u(\cdot, t)\right\|_{\infty}=0, \quad \text { almost surely. }
$$

Remark 2.2. By A1, the parameter $\ell$ represents the order of the initial quantity of particles per site. Condition A2 states that the growth of $\ell$ cannot be too slow in comparison with $N$. For instance, $\ell(N)=N^{\varepsilon}$ satisfies A2 for any $\varepsilon>0$.

An immediate corollary of Theorem 2.1 is the following

Corollary 2.3. If $\mathrm{A} 1$ and $\mathrm{A} 2$ hold, we have

$$
\liminf _{N \rightarrow \infty} T_{\max }^{N} \geq T_{\max } \quad \text { almost surely. }
$$

The left hand side in the above equation can be infinity in general. Next we find conditions to guarantee that this is not the case.

Proposition 2.4. Let $b \in C^{1}$ be convex, positive and such that $\int_{0}^{\infty} 1 / b(s) d s<+\infty$. Assume also that $d$ is bounded or linear, then for every $N$ we have

$$
\mathbb{P}\left(T_{\max }^{N}<+\infty\right)=1 .
$$

Conditions on the growth of $b$ cannot be removed as can be shown with simple examples. The convexity assumption is technical, but we are not able to remove it. It can be weakened to some extent assuming that $b$ is convex on some interval of the form $[a,+\infty)$. Finally, we obtain

Theorem 2.5. Assume $d$ is bounded or linear and $b(s)=s^{p}+d(s)$ with $1<p \leq 3$. If A1 and $\mathrm{A} 2$ hold, then

$$
\lim _{N \rightarrow \infty} T_{\max }^{N}=T_{\max }, \quad \text { in probability. }
$$

The paper is organized as follows: in Section 3 we give a graphical construction of the process. Section 4 deals with a semidiscrete approximation of equation (1). We discretize the space variable and prove that the solution of the ODE obtained with this procedure converges to $u$ as the mesh parameter goes to zero. In Section 5 we prove Theorem 2.1. We first prove this theorem for birth rates $b$ with compact support relying on results of Blount [4] and then we extend these results to the general case that allows explosions by means of truncation and 
a coupling argument. Finally, in Section 6 we study the asymptotic behavior of the particle system. We prove that the process explodes with probability one, Proposition 2.4 and that the blow-up times converges to the explosion time of the PDE (1), Theorem 2.5.

\section{Graphical construction}

In this section we give the so called Harris graphical construction of the process: we construct the particle system as a deterministic function of a family of Poisson processes. This construction will be useful on the one hand to couple processes with different birth rates $b$ and on the other hand to construct jointly a birth and death process that bounds the total number of particles in the system from below.

Let $\left(\mathcal{N}^{+}(i), \mathcal{N}^{-}(i), i \geq 1\right)$ be a family of Poisson processes in $\mathbb{R}_{+}$with rate $N^{2}$. Let also $\left(\mathcal{N}_{b}(r, k), \mathcal{N}_{d}(r, k), r \geq 1,1 \leq k \leq N\right)$ be a family of Poisson processes in $\mathbb{R}_{+}$with rates $\ell b\left(\ell^{-1} r\right), \ell d\left(\ell^{-1} r\right)$ respectively. All the processes are taken independent. We construct a process $\xi(t)=\left(\xi_{1}(t), \xi_{2}(t) \ldots\right)$ that determines the position of each particle and the process $\eta(t)$ is defined as the empirical measure of $\xi(t)$, i.e.

$$
\eta_{k}(t)=\sum_{i=1}^{K(t)} \mathbf{1}\left\{\xi_{i}(t)=k\right\} .
$$

The variable $K(t)$ is the total number of particles in the system and will be defined inductively jointly with the construction of the process. Initially we start with $K(0)$ labeled particles $1, \ldots, K(0)$ at positions $\xi_{1}, \ldots, \xi_{K(0)}$. Assume the process is defined up to time $s \geq 0$ and proceed by recurrence. Start with $s=0$ and define

$$
\tau(s, \xi(s)):=\inf \left\{t>s: t \in \cup_{i=1}^{K(s)}\left(\mathcal{N}^{+}(i) \cup \mathcal{N}^{-}(i)\right) \cup \cup_{k \in \mathbb{T}_{N}} \mathcal{N}_{b}\left(\eta_{k}(s), k\right) \cup \mathcal{N}_{d}\left(\eta_{k}(s), k\right)\right\} .
$$

For $t \in[s, \tau)$ define $\xi(t)=\xi(s)$ and then

1. If $\tau \in \mathcal{N}^{ \pm}(i)$ define $\xi_{i}(\tau)=\xi_{i}(s) \pm 1$ and $\xi_{j}(\tau)=\xi_{j}(s)$ if $j \neq i$.

2. If $\tau \in \mathcal{N}_{b}\left(\eta_{k}(s), k\right)$ set $\xi_{K(s)+1}(\tau)=k ; K(\tau)=K(s)+1$ and $\xi_{i}(\tau)=\xi_{i}(s)$ for $1 \leq i \leq K(s)$.

3. If $\tau \in \mathcal{N}_{d}\left(\eta_{k}(s), k\right)$, let $j:=\min \left\{i: \xi_{i}(s)=k\right\}$. Set $\xi_{j}(\tau)=\xi_{K(s)}(s) ; K(\tau)=K(s)-1$ and for $i \neq j, 1 \leq i \leq K(s)-1$ define $\xi_{i}(\tau)=\xi_{i}(s)$.

In words, if $\tau \in\left(\mathcal{N}^{+}(i) \cup \mathcal{N}^{-}(i)\right)$ (where $i$ is a particle in the system), then this particle moves to the right or to the left according to wether $\tau \in \mathcal{N}^{+}(i)$ or $\tau \in \mathcal{N}^{-}(i)$. If $\tau \in \mathcal{N}_{b}\left(\eta_{k}(s)\right)$, a new particle is created at site $k$, and hence $K(s)$ increases in one. Finally, if $\tau \in \mathcal{N}_{d}\left(\eta_{k}(s)\right)$, a particle is killed at site $k$. We kill the particle with minimum index and we assign this index to the particle with index $K(s)$ (and then decrease $K(s)$ in one), so that the alive particles are always the ones with index $1, \ldots, K(\cdot)$. The process is then defined up to time $\tau$. Put $s=\tau$ and iterate to define $\xi(t)$ and $\eta(t)$ up to time $t=T_{\max }^{N}$. Observe that $T_{\max }^{N}<\infty$ if and only if $\eta\left(T_{\max }^{N}\right):=\lim _{t \nearrow T_{\max }^{N}}\|\eta(t)\|_{\infty}=\infty$, and in this case, the sum of the waiting times is summable and equal to $T_{\max }^{N}$. 
Coupling processes with different birth rates. Observe that if we want to construct two different copies $\eta, \tilde{\eta}$ of the above process with two different birth rates $b, \tilde{b}$ and we have $b(s)=\tilde{b}(s)$ for $s \leq M$ then we can couple them by using the same Poisson processes $\mathcal{N}^{+}(i), \mathcal{N}^{-}(i), i \geq 1, \overline{\mathcal{N}}_{d}(r, \cdot), r \geq 1$ and $\mathcal{N}_{b}(r, \cdot)$ for $r \leq M$. In this way we obtain that almost surely $\eta(t)=\tilde{\eta}(t)$ for $0 \leq t \leq T_{M}^{N}$, the first time that the process reaches the value $M$.

\section{Convergence of a semidiscrete scheme}

We now consider deterministic spatial discretizations of (1), keeping continuous the time variable. The goal is to prove convergence of such spatial discretizations to the solution $u$ of the partial differential equation (1). This result will be used as an intermediate step in the proof of the Theorem 2.1.

Throughout this section, we assume that the function $f$ in (1) is globally Lipschitz.

We define the semidiscrete approximation $u^{N}(t)=\left(u_{1}^{N}(t), \ldots, u_{N}^{N}(t)\right)$ of the PDE (1) as the solution of the following ODE system:

$$
\begin{cases}\frac{d}{d t} u_{k}^{N}(t)=N^{2}\left[u_{k+1}^{N}(t)-2 u_{k}^{N}(t)+u_{k-1}^{N}(t)\right]+f\left(u_{k}^{N}(t)\right), & k \in \mathbb{T}_{N}, \\ u_{k}^{N}(0)=\varphi\left(x_{k}\right), & k \in \mathbb{T}_{N} .\end{cases}
$$

Proposition 4.1. Let $u \in C^{4,1}(\mathbb{T} \times[0, T])$ be a positive solution of $(1)$ and $u^{N}(t)$ the semidiscrete approximation given by (5). Then, there exists a positive constant $C$ depending on the $C^{4,1}(\mathbb{T} \times[0, T])$ norm of $u$ such that, for every $N$ large enough,

$$
\sup _{t \in[0, T]} \max _{k \in \mathbb{T}_{N}}\left|u\left(x_{k}, t\right)-u_{k}^{N}(t)\right| \leq C N^{-2} .
$$

As a consequence of Proposition 4.1, if $u \in C^{4,1}(\mathbb{T} \times[0, T])$ then

$$
\limsup _{N \rightarrow \infty} \sup _{t \in[0, T]} \max _{k \in \mathbb{T}_{N}}\left|u_{k}^{N}(t)\right|<\infty .
$$

We need the next lemma about solutions (and supersolutions) of the following ODE system

$$
\begin{cases}z_{k}^{\prime}=N^{2}\left(z_{k+1}-2 z_{k}+z_{i-1}\right)+C_{*}\left(\left|z_{k}\right|+N^{-2}\right), & k \in \mathbb{T}_{N}, \\ z_{k}(0)=0, & k \in \mathbb{T}_{N} .\end{cases}
$$

Definition 4.1. We say that $\bar{Z}=\left(\bar{z}_{1}, \ldots, \bar{z}_{N}\right)$ is a supersolution of $(7)$ if

$$
\begin{cases}\bar{z}_{k}^{\prime} \geq N^{2}\left(\bar{z}_{k+1}-2 \bar{z}_{k}+\bar{z}_{k-1}\right)+C_{*}\left(\left|\bar{z}_{k}\right|+N^{-2}\right), & k \in \mathbb{T}_{N}, \\ \bar{z}_{k}(0) \geq 0, & k \in \mathbb{T}_{N} .\end{cases}
$$

Analogously, we say that $\underline{Z}$ is a subsolution if it satisfies (8) with the reverse inequalities.

Lemma 4.2. Let $\bar{Z}$ and $\underline{Z}$ be a supersolution and a subsolution of (8) respectively, and let $Z$ be a solution of (7). Then

$$
\bar{Z}(t) \geq Z(t) \geq \underline{Z}(t) .
$$

Proof. By an approximation procedure we restrict ourselves to consider strict inequalities in (8). If that is not the case, we consider $\tilde{Z}(t)=\bar{Z}(t)+C \varepsilon t$ with adequate $C$, and letting $\varepsilon \searrow 0$ yields the result. 
We prove that $\bar{Z}(t)>Z(t)$ arguing by contradiction. Assume that there exists a first time $t_{*}$ and $k \in \mathbb{T}_{N}$ such that $\bar{z}_{k}\left(t_{*}\right)=z_{k}\left(t_{*}\right)$. Then, we would have

$$
0 \geq \bar{z}_{k}^{\prime}\left(t_{*}\right)-z_{k}^{\prime}\left(t_{*}\right)>N^{2}\left(\bar{z}_{k+1}\left(t_{*}\right)-z_{k+1}\left(t_{*}\right)+\bar{z}_{k-1}\left(t_{*}\right)-z_{k-1}\left(t_{*}\right)\right) \geq 0,
$$

a contradiction. The inequality $Z(t) \geq \underline{Z}(t)$ is handled in a similar way.

Proof of Proposition 4.1. For $k \in \mathbb{T}_{N}$ denote $u_{k}=u\left(x_{k}, t\right)$ and define the error function

$$
e_{k}:=u_{k}^{N}-u_{k} \text {. }
$$

By means of Taylor's expansion, for $k \in \mathbb{T}_{N}$, there exist $c_{k} \in\left(x_{k}, x_{k+1}\right)$ and $\tilde{c}_{k} \in\left(x_{k-1}, x_{k}\right)$ such that

$$
u_{k+1}=u_{k}+u_{x}\left(x_{k}, t\right) \frac{1}{N}+u_{x x}\left(x_{k}, t\right) \frac{1}{2 ! N^{2}}+u_{x x x}\left(x_{k}, t\right) \frac{1}{3 ! N^{3}}+u_{x x x x}\left(c_{k}, t\right) \frac{1}{4 ! N^{4}}
$$

and

$$
u_{k-1}=u_{k}-u_{x}\left(x_{k}, t\right) \frac{1}{N}+u_{x x}\left(x_{k}, t\right) \frac{1}{2 ! N^{2}}-u_{x x x}\left(x_{k}, t\right) \frac{1}{3 ! N^{3}}+u_{x x x x}\left(\tilde{c}_{k}, t\right) \frac{1}{4 ! N^{4}} .
$$

Summing the equations above and recalling that $u$ is the solution of (1) gives

$$
u_{k}^{\prime}=N^{2}\left(u_{k+1}-2 u_{k}+u_{k-1}\right)+f\left(u_{k}\right)-\frac{1}{4 ! N^{2}}\left(u_{x x x x}\left(\tilde{c}_{k}\right)+u_{x x x x}\left(c_{k}\right)\right) .
$$

Writing $a_{k}:=\frac{1}{4 !}\left(u_{x x x x}\left(\tilde{c}_{k}\right)+u_{x x x x}\left(c_{k}\right)\right)$, we get that the error function satisfies, for $k \in \mathbb{T}_{N}$,

$$
e_{k}^{\prime}=N^{2}\left(e_{k+1}-2 e_{k}+e_{k-1}\right)+f\left(u_{k}^{N}\right)-f\left(u_{k}\right)-a_{k} N^{-2} .
$$

Since $f$ is globally Lipschitz, there exists a positive constant $C_{*}$ independent of $N$ such that

$$
e_{k}^{\prime} \leq N^{2}\left(e_{k+1}-2 e_{k}+e_{k-1}\right)+C_{*}\left(\left|e_{k}\right|+N^{-2}\right), \quad \forall k \in \mathbb{T}_{N}
$$

Hence $\left(e_{1}, \ldots, e_{N}\right)$ is a sub-solution of (8). Consider the super-solution $\bar{Z}=\left(\bar{z}_{1}, \ldots, \bar{z}_{N}\right)$ given by $\bar{z}_{k}(t)=e^{2 C_{*} t} / N^{2}$. Notice that $\bar{Z}$ verifies (8). By Lemma 4.2,

$$
e_{i}(t) \leq \bar{z}_{i}(t) \leq e^{2 C_{*} T} / N^{2} \quad \text { for all } k \in \mathbb{T}_{N} .
$$

Repeating the same arguments as before with $-e_{i}$, we obtain

$$
\left|e_{k}(t)\right| \leq \bar{z}_{k}(t) \leq e^{2 C_{*} T} / N^{2} \quad \text { for all } k \in \mathbb{T}_{N} .
$$

This completes the proof.

\section{LIMIT FOR THE DENSITY OF PARTICLES.}

The following key estimate is obtained by Blount in [4].

Theorem 5.1 (Blount, [4]). Assume $b$ is Lipschitz continuous with compact support and fix $T>0$. There exist constants $K, a>0$ depending on $T$ and $\varphi$ and a process $Y^{N}$ such that

$$
\left\|\bar{X}^{N}(t)-u^{N}(t)\right\|_{\infty} \leq\left(1+K t e^{K t}\right)\left(K\left\|\bar{X}^{N}(0)-u^{N}(0)\right\|_{\infty}+\sup _{s \in[0, t]}\left\|Y^{N}(s)\right\|_{\infty}\right)
$$

for all $0 \leq t \leq T$. Moreover, for every $\varepsilon>0$ we have

$$
\mathbb{P}\left(e^{-4 T} \sup _{t \in[0, T]}\left\|Y^{N}(t)\right\|_{\infty}>\varepsilon\right) \leq 4 N^{3} e^{-a \varepsilon^{2} \ell} .
$$

The proof in [4] considers the case where $f$ is a polynomial with negative leading term, but the proof can be extended to our case with no difficulty. 
Proof of Theorem 2.1. Assume first that $b$ has compact support. Condition (A2) in Theorem 2.1 means that the bound in (9) is summable in $N$, so Borel-Cantelli's lemma implies $\sup _{t \in[0, T]}\left\|\bar{X}^{N}(t)-u^{N}(t)\right\|_{\infty} \rightarrow 0$ almost surely. This fact, combined with Proposition 4.1 gives us

$$
\lim _{N \rightarrow \infty} \sup _{t \in[0, T]}\left\|\bar{X}^{N}(t)-u(\cdot, t)\right\|_{\infty}=0, \quad \text { almost surely }
$$

if $b$ has compact support.

For general $b$, we consider

$$
M=\sup _{t \in[0, T]}\|u(\cdot, t)\|_{\infty}
$$

which is finite since we are imposing $T<T_{\max }$.

Let $b_{M+1}$ be a smooth function with compact support that coincides with $b$ in the interval $[0, M+1]$. Denote by $X^{N, M+1}(x, t)$ the process with creation of particles driven by $b_{M+1}$ instead of $b$.

By making use of Harris graphical construction of Section 3, for each $N$ we can couple the processes $X^{N, M+1}(x, t)$ and $X^{N}(x, t)$ in such a way that their trajectories coincide up to the stopping time

$$
T_{M+\frac{1}{2}}^{N}=\inf \left\{t \geq 0 ;\left\|X^{N, M+1}(t)\right\|_{\infty} \geq M+\frac{1}{2}\right\}
$$

Observe that

$$
\left\|X^{N}(t)-u(\cdot, t)\right\|_{\infty} \leq\left\|X^{N}(t)-X^{N, M+1}(t)\right\|_{\infty}+\left\|X^{N, M+1}(t)-u(\cdot, t)\right\|_{\infty}
$$

Denote by $u^{M+1}(\cdot, t)$ the solution of (1) with $f=b_{M+1}-d$. Since uniqueness hold for (1) and $\|u(\cdot, t)\|_{\infty} \leq M$ in $[0, T]$, we have $u^{M+1}(\cdot, t)=u(\cdot, t)$, for $t \in[0, T]$. Thus, the second term in (11) can be replaced by $\left\|X^{N, M+1}(t)-u^{M+1}(t)\right\|_{\infty}$, which goes to zero as $N \uparrow \infty$ uniformly and almost surely. Hence there exists a finite random $N_{0}$ such that, for $N \geq N_{0}$,

$$
\sup _{t \in[0, T]}\left\|X^{N, M+1}(t)\right\|_{\infty} \leq \sup _{t \in[0, T]}\|u(\cdot, t)\|_{\infty}+\frac{1}{2}=M+\frac{1}{2} .
$$

Since $X^{N}$ and $X^{N, M+1}$ are coupled, the first term on the r.h.s of (11) vanishes for $N \geq N_{0}$, concluding the proof of the theorem.

Observe that for $\varepsilon>0$, Theorem 2.1 implies $\lim \inf _{N \rightarrow \infty} T_{\operatorname{expl}}(N) \geq T_{\max }-\varepsilon$ almost surely. Letting $\varepsilon \searrow 0$ yields

$$
\liminf _{N \rightarrow \infty} T_{\operatorname{expl}}(N) \geq T_{\max }, \quad \text { almost surely, }
$$

proving Corollary 2.3.

\section{The EXPlosion times}

In this section we prove the finiteness of the explosion time $T_{\max }^{N}$ for every $N$, Proposition 2.4 and the convergence as $N \rightarrow \infty$, Theorem 2.5. Both proofs rely on a coupling with a one-dimensional birth and death process.

The goal is to construct (jointly with $X^{N}$ ) a process $Y=(Y(t), t \geq 0$ ) that dominates the total number of particles $\|\eta(t)\|_{\infty}:=\sum_{k} \eta_{k}(t)$ from below almost surely and for every time. According to the model, the rate at which a new particle (somewhere) is created is 
given by $\sum_{k \in \mathbb{T}_{N}} \ell b\left(\frac{\eta_{k}}{\ell}\right)$, the sum of the rates of each individual site. Since $b$ is convex and nondecreasing,

$$
\sum_{k \in \mathbb{T}_{N}} \ell b\left(\frac{\eta_{k}}{\ell}\right)=\ell N \sum_{k \in \mathbb{T}_{N}} \frac{1}{N} b\left(\frac{\eta_{k}}{\ell}\right) \geq \ell N b\left(\frac{1}{\ell N} \sum_{i \in \mathbb{T}_{N}} \eta_{i}\right) \geq \ell N b\left(\frac{|\eta|}{\ell N}\right)=: q(|\eta|,|\eta|+1) .
$$

Analogously, if $d$ is bounded, the rate for annihilation of a particle is the sum of the rates in each site, which we bound (for $\eta \neq 0$ ) by

$$
\sum_{k \in \mathbb{T}_{N}} \ell d\left(\frac{\eta_{k}}{\ell}\right) \leq \ell N\|d\|_{\infty}=: q(|\eta|,|\eta|-1) .
$$

Then, we can construct a process $(Y(t))$ with rates $q$ jointly with $(\eta(t))$, in such a way that $\|\eta(t)\|_{\infty} \geq Y(t)$ almost surely. We need to slightly modify the construction of $\eta(t)$. The construction is almost the same with the only modification that for each $k$, we construct the Poisson processes $\mathcal{N}_{d}(r, k), r \geq 1$ as independent thinnings of a Poisson process $\mathcal{N}_{d}(k)$ with rate $\ell\|d\|_{\infty}$ and we add (independent) uniform marks to the points of the Poisson processes $\mathcal{N}_{b}(r, k)$, i.e.: to each point $t \in \mathcal{N}_{b}(r, k)$ we attach a random variable $U_{t}$ with uniform distribution in $[0,1]$. All of them independent of all the processes. We construct $Y$ recursively. Assume that the process is defined up to time $s$. Let

$$
\tau(s, Y(s)):=\inf \left\{t>s: t \in \cup_{k \in \mathbb{T}_{N}} \mathcal{N}_{b}\left(\eta_{k}(s), k\right) \cup \mathcal{N}_{d}(k)\right\} .
$$

For $t \in[s, \tau(s, Y(s)))$ define $Y(t)=Y(s)$ and then

1. If $\tau \in \cup_{k \in \mathbb{T}_{N}} \mathcal{N}_{b}\left(\eta_{k}(s), k\right)$ and $U_{\tau(s, Y(s))}<\ell N b\left(\frac{|\eta|}{\ell N}\right) / \sum_{k \in \mathbb{T}_{N}} \ell b\left(\frac{\eta_{k}}{\ell}\right)$, set $Y(\tau)=$ $Y(s)+1$.

2. If $\tau \in \cup_{k \in \mathbb{T}_{N}} \mathcal{N}_{d}(k)$, set $Y(\tau)=Y(s)-1$.

3. Otherwise set $Y(\tau)=Y(s)$.

The process is then defined up to time $\tau$. Put $s=\tau$ and iterate to define $Y(t)$ as long as possible. This construction guarantees that if for some time $s \geq 0$ we have $\|\eta(s)\|_{\infty} \geq Y(s)$ then

$$
\|\eta(t)\|_{\infty} \geq Y(t), \quad \text { for all } t \geq s .
$$

It is straightforward to check that in addition, $Y$ is a birth and death process with rates $q(r, r+1)=\ell N b(r / \ell N)$, and $q(r, r-1)=\ell N\|d\|_{\infty}$. The next step is to proof that $Y$ explodes in finite time and estimate the explosion time of $Y(t)$. We begin with some considerations about birth and death processes with rates $\mathbf{b}_{r}$ and $\mathbf{d}_{r}$ respectively. For such a process, let $\tau_{r}$ be the hitting time of the state $r \in \mathbb{N}$ and denote

$$
f_{r}=\mathbb{E}_{r}\left[\tau_{r+1}\right]
$$

the expected time to hit $r+1$, starting at $r$. By the Markov property,

$$
f_{r+1}=\frac{1}{\mathbf{b}_{r}+\mathbf{d}_{r}}+\frac{\mathbf{d}_{r}}{\mathbf{b}_{r}} f_{r}, \quad \text { for } r \geq 0 .
$$

Notice that $\sum_{r=r_{0}}^{\infty} f_{r}$ is the expected time spent by the process starting from $r_{0} \in \mathbb{N}$ before reaching $\infty$. With an inductive procedure we derive the formula

$$
\sum_{r=1}^{n} f_{r}=\sum_{r=0}^{n-1} \frac{1}{\mathbf{b}_{r}+\mathbf{d}_{r}}+f_{0} \sum_{r=0}^{n-1} \prod_{j=0}^{r} \frac{\mathbf{d}_{j}}{\mathbf{b}_{j}}+\sum_{r=0}^{n-2} \frac{1}{\mathbf{b}_{r}+\mathbf{d}_{r}} \sum_{j=r+1}^{n-1} \prod_{i=r}^{j} \frac{\mathbf{d}_{i}}{\mathbf{b}_{i}} .
$$


We invoke now that $\mathbf{b}_{r}=\ell N b\left(\frac{r}{\ell N}\right), \mathbf{d}_{r}=\ell N\|d\|_{\infty}$. Notice also that

$$
\int_{1}^{\infty} \frac{d s}{b(s)}<\infty
$$

If $d$ is bounded (or linear), then $\mathbf{b}_{r} / \mathbf{d}_{r} \rightarrow 0$ as $r \rightarrow \infty$ and hence the three terms in (15) are finite as $n \rightarrow \infty$. The first one behaves as $\int 1 / b$, each term of the sum in the second term can be bounded by $C e^{-r}$ and using these two facts, we bound the third term. We have proved Proposition 2.4 for $d$ bounded. Observe that if $d$ is not bounded but linear, equation (13) takes the form

$$
\sum_{k \in \mathbb{T}_{N}} \ell d\left(\frac{\eta_{k}}{\ell}\right)=d(|\eta|)=: q(|\eta|,|\eta|-1) .
$$

Hence we don't need the thinnings to couple $Y$ and $\eta$. Both processes move jointly to the left always. The rest of the proof follows along the same steps.

The proof of Theorem 2.5 is based on a more delicate analysis of (15), but we first need some knowledge on the solutions of (1). That is the context of the following theorem.

Theorem 6.1. Assume $f$ is nonnegative and continuously differentiable.

(1) There exists a time $T_{\max }>0$ (possibly infinite) such that there exists a unique maximal solution to (1) in $\left[0, T_{\max }\right)$.

(2) If $T_{\max }<+\infty$ we have

$$
\lim _{t \nearrow T_{\max }}\|u(t, \cdot)\|_{L^{\infty}(\mathbb{T})}=+\infty .
$$

(3) If $f$ is convex and positive for $r \geq r_{0}$ and $\varphi(x) \geq r_{0}$ for all $x \in \mathbb{T}$, then $u$ blows up in finite time $T_{\max }$ and moreover the following estimate holds

$$
T_{\max } \leq \int_{\|\varphi\|_{L^{1}(\mathbb{T})}}^{\infty} \frac{1}{f(s)} d s .
$$

(4) If $f(u)=u^{p}$ with $1<p \leq 3$, then

$$
\lim _{t \nearrow T_{\max }}\|u(t, \cdot)\|_{L^{1}(\mathbb{T})}=+\infty .
$$

Proof. Existence and uniqueness of a smooth classical (maximal) solution up to time $T_{\max }$ is proved in [18]. See also [5, 13, 14, 15].

To prove (2) consider $y$, the solution of the ODE $y^{\prime}=f(y), y(0)=\|\varphi\|_{L^{\infty}(\mathbb{T})}$. By comparison arguments, we get $u(x, t) \leq y(t)$ for every $t$ and hence the blow-up time $T_{\max }=T_{\max }(\varphi)$ is bigger than the one for $y$. So, assume

$$
\limsup _{t \nearrow T_{\max }}\|u(t, \cdot)\|_{L^{\infty}(\mathbb{T})} \leq C .
$$

Then, there is an increasing sequence of times $\left(t_{k}\right)$ such that $t_{k} \nearrow T_{\max }$ and such that the solution to $(1)$ with initial data $u\left(\cdot, t_{k}\right)$ is defined in some interval $[0, \tilde{T}]$, where $\tilde{T}$ depends only on $C$ (and not on $k$ ). Then we can extend $u$ for times $t>T_{\max }$ which contradicts (1).

For (3) consider $\Phi(t):=\|u(\cdot, t)\|_{L^{1}(\mathbb{T})}$. Observe that $v(x, t)=\varphi(x)$ verifies $v_{t} \leq v_{x x}+f(v)$ and then the solution $u$ with initial data $\varphi$ verifies $u(x, t) \geq v(t, x)=\varphi(x)$ for every $x \in \mathbb{T}$, $t \geq 0$. Differentiating and using Jensen's inequality we get

$$
\frac{d}{d t} \Phi(t)=\int_{\mathbb{T}} f(u(x, t)) d x \geq f(\Phi(t)), \quad \Phi(0)=\|\varphi\|_{L^{1}(\mathbb{T})} .
$$


Thus, $\Phi(t) \geq z(t)$, the solution of the $\mathrm{ODE} z^{\prime}=f(z), z(0)=\|\varphi\|_{L^{1}(\mathbb{T})}$. Integrating this equation we obtain

$$
\int_{z(0)}^{z(t)} \frac{1}{f(s)} d s \geq t
$$

Let $T_{z}$ be the maximal existence time for $z$. Taking the limit $t \nearrow T_{z}$, we first observe that $T_{z}<\infty$ and $z\left(T_{z}\right)=+\infty$, and next

$$
T_{\max } \leq T_{z} \leq \int_{\|\varphi\|_{L^{1}(\mathbb{T})}}^{\infty} \frac{1}{f(s)} d s .
$$

For (4) we use the following dichotomy proved by Velázquez [17]. Assume that $u(x, t)$ is a positive solution to $u_{t}=u_{x x}+u^{p}$ for $x \in(-R, R)$ and $t \in(0, T)$ which blows up at $t=T$. Assume also that its blow-up set

$$
B=\left\{\bar{x} \in(-R, R): \limsup _{t \nearrow T}\|u(t, \cdot)\|_{L^{\infty}(\mathbb{T})}=+\infty\right\}
$$

is contained in $[-\delta, \delta]$ for some $\delta<R$. Then $B$ is isolated and for any blow-up point $\bar{x} \in B$ one of the following holds.

$$
\begin{aligned}
& \lim _{x \rightarrow \bar{x}}\left(\frac{|x-\bar{x}|^{2}}{|\log | x-\bar{x}||}\right)^{\frac{1}{p-1}} u\left(x, T_{\max }\right)=\left(\frac{8 p}{(p-1)^{2}}\right)^{\frac{1}{p-1}}, \\
& \lim _{x \nearrow_{\bar{x}}}|x-\bar{x}|^{\frac{m}{p-1}} u\left(x, T_{\max }\right)=((p-1) C)^{-\frac{1}{p-1}},
\end{aligned}
$$

where $C$ and $m$ are positive constants with $m \geq 4$. Also Chen and Matano proved [6] that if the initial data $\varphi$ is non-constant, the number of blow-up points of (1) is finite (moreover, it does not exceed the number of local maximum of $\varphi$ ) and hence we can apply the above dichotomy in a neighborhood of a blow-up point. Fatou's lemma and straightforward computations leads to $\liminf _{t \nearrow T}\|u(\cdot, t)\|_{L^{1}(\mathbb{T})} \geq\|u(\cdot, T)\|_{L^{1}(\mathbb{T})}=+\infty$ if $1<p \leq 3$ for any of the alternatives. If $\varphi$ is constant, the conclusion is immediate and holds for every $p>1$.

Proof of Theorem 2.5. If $T_{\max }=\infty$, Corollary 2.3 implies the theorem. We observe that this only happens if $f$ has a root at $r_{0}$ and $\varphi \equiv r_{0}$. For $T_{\max }<\infty$, we assume $d$ is bounded (if $d$ is not bounded but linear, the proof is similar). From (15) we get

$$
\begin{aligned}
\sum_{r=y \ell N}^{n} f_{r} & =\sum_{r=y \ell N}^{n-1} \frac{1}{\mathbf{b}_{r}+\mathbf{d}_{r}}+f_{0} \sum_{r=y \ell N}^{n-1} \prod_{j=0}^{r} \frac{\mathbf{d}_{j}}{\mathbf{b}_{j}}+\sum_{r=y \ell N}^{n-2} \frac{1}{\mathbf{b}_{r}+\mathbf{d}_{r}} \sum_{j=r+1}^{n-1} \prod_{i=r}^{j} \frac{\mathbf{d}_{i}}{\mathbf{b}_{i}} \\
& \leq \frac{1}{\ell N} \sum_{r=y \ell N}^{n-1} \frac{1}{b(r / \ell N)}+f_{0} C \sum_{r=y \ell N}^{n-1} e^{-r}+\frac{1}{\ell N} \sum_{r=y \ell N}^{n-2} \frac{C}{b(r / \ell N)} \sum_{j=r+1}^{n-1} e^{-(j-r)} \\
& \leq \int_{y}^{\infty} \frac{1}{b(s)} d s+C^{\prime} e^{-\frac{y \ell N}{2}}+C^{\prime \prime} \int_{y}^{\infty} \frac{1}{b(s)} d s .
\end{aligned}
$$

Since there exists $r_{0}$ such that $\mathbf{d}_{r} / \mathbf{b}_{r}<e^{-1}$ for $r \geq r_{0}$ and the indexes in the sums start at $r=y \ell N$, the constants $C, C^{\prime}, C^{\prime \prime}$ are independent of $\ell$ and $N$.

Fix $\delta>0$ and choose $M$ large enough in order to guarantee that

$$
\int_{M}^{\infty} \frac{1}{b(s)} d s+C^{\prime} e^{-\frac{M}{2}}+C^{\prime \prime} \int_{M}^{\infty} \frac{1}{b(s)} d s<\delta .
$$


Observe that $M$ does not depend on $N$. By Theorem 6.1, there exists a time $T<T_{\max }$ such that

$$
\|u(\cdot, T)\|_{L^{1}(\mathbb{T})} \geq M+1 .
$$

Take $\varepsilon=1$ and apply Theorem 2.1, to get the existence of a finite random $N_{0} \in \mathbb{N}$ such that, for $N \geq N_{0},\left\|X^{N}(T, \cdot)-u(T, \cdot)\right\|_{\infty}<1$, which implies $\left\|X^{N}(T, \cdot)\right\|_{L^{1}(\mathbb{T})}>M$. Hence,

$$
\sum_{k \in \mathbb{T}_{N}} \eta_{k}(T) \geq M \ell N
$$

For each $N$ and from time $T$ on we construct the process $Y$, with initial data $Y(T)=M \ell N$ jointly with $\eta(t)$. Using that the expected time for the explosion time of the process $Y$ is given by

$$
T+\sum_{r=M \ell N}^{\infty} f_{r}
$$

and (16) we get that the explosion time of $Y, T_{Y}$, has expectation bounded by $T+\delta$ and that for $N \geq N_{0}$, this time bounds from above the explosion time $T_{\max }^{N}$ of $\eta$. Hence, for $\gamma>0$ we have

$$
\begin{aligned}
\mathbb{P}\left(T_{\max }^{N}>T_{\max }+\gamma\right) & \leq \mathbb{P}\left(T_{\max }^{N}>T_{\max }+\gamma, N>N_{0}\right)+\mathbb{P}\left(T_{\max }^{N}>T_{\max }+\gamma, N \leq N_{0}\right) \\
& \leq \mathbb{P}\left(T_{Y}>T+\gamma\right)+\mathbb{P}\left(N \leq N_{0}\right) .
\end{aligned}
$$

Hence, the finiteness of $N_{0}$ and Markov inequality gives us

$$
\limsup _{N \rightarrow \infty} \mathbb{P}\left(T_{\max }^{N}>T_{\max }+\gamma\right) \leq \frac{\delta}{\gamma} .
$$

Since $\delta$ is arbitrary and Corollary 2.3 implies the reversed inequality, the proof is completed.

\section{ACKNOWLEDGEMENTS}

We want to thank Pablo Ferrari, Milton Jara and Mariela Sued for fruitful discussions.

PG is partially supported by UBACyT 20020090100208, ANPCyT PICT No. 2008-0315 and CONICET PIP 2010-0142 and 2009-0613 and MathAmSud project "Stochastic structure of large interacting systems".

\section{REFERENCES}

[1] L. Arnold and M. Theodosopulu. Deterministic limit of the stochastic model of chemical reactions with diffusion. Adv. in Appl. Probab., 12(2):367-379, 1980.

[2] Catherine Bandle and Hermann Brunner. Blowup in diffusion equations: a survey. J. Comput. Appl. Math., 97(1-2):3-22, 1998.

[3] Douglas Blount. Comparison of stochastic and deterministic models of a linear chemical reaction with diffusion. The Annals of Probability, 19(4):1440-1462, 1991.

[4] Douglas Blount. Law of large numbers in the supremum norm for a chemical reaction with diffusion. The Annals of Applied Probability, 2(1):131-141, 1992.

[5] Xu-Yan Chen. Uniqueness of the $\omega$-limit point of solutions of a semilinear heat equation on the circle. Proc. Japan Acad. Ser. A Math. Sci., 62(9):335-337, 1986.

[6] Xu-Yan Chen and Hiroshi Matano. Convergence, asymptotic periodicity, and finite-point blow-up in onedimensional semilinear heat equations. J. Differential Equations, 78(1):160-190, 1989.

[7] Victor A. Galaktionov and Juan L. Vázquez. The problem of blow-up in nonlinear parabolic equations. Discrete Contin. Dyn. Syst., 8(2):399-433, 2002. Current developments in partial differential equations (Temuco, 1999). 
[8] Claude Kipnis and Claudio Landim. Scaling limits of interacting particle systems. Grundlehren der mathematischen Wissenschaften ; 320. Springer, 1999.

[9] Peter Kotelenez. Law of large numbers and central limit theorem for linear chemical reactions with diffusion. The Annals of Probability, 14(1):173-193, 1986.

[10] Peter Kotelenez. High density limit theorems for nonlinear chemical reactions with diffusion. Probability theory and related fields, 1988.

[11] Mustapha Mourragui. Hydrodynamic limit for a jump, birth and death process. (Limite hydrodynamique d'un processus de sauts, de naissances et de morts.). 1993.

[12] J. R. Norris. Markov Chains (Cambridge Series in Statistical and Probabilistic Mathematics). Cambridge University Press, July 1998.

[13] C. V. Pao. Nonlinear parabolic and elliptic equations. Plenum Press, New York, 1992.

[14] Pavol Quittner and Philippe Souplet. Superlinear parabolic problems. Birkhäuser Advanced Texts: Basler Lehrbücher. [Birkhäuser Advanced Texts: Basel Textbooks]. Birkhäuser Verlag, Basel, 2007. Blow-up, global existence and steady states.

[15] Alexander A. Samarskii, Victor A. Galaktionov, Sergei P. Kurdyumov, and Alexander P. Mikhailov. Blow-up in quasilinear parabolic equations, volume 19 of de Gruyter Expositions in Mathematics. Walter de Gruyter \& Co., Berlin, 1995. Translated from the 1987 Russian original by Michael Grinfeld and revised by the authors.

[16] Juan Luis Vázquez. The porous medium equation. Oxford Mathematical Monographs. The Clarendon Press Oxford University Press, Oxford, 2007. Mathematical theory.

[17] J. J. L. Velázquez. Local behaviour near blow-up points for semilinear parabolic equations. J. Differential Equations, 106(2):384-415, 1993.

[18] Fred B. Weissler. Semilinear evolution equations in Banach spaces. J. Funct. Anal., 32(3):277-296, 1979.

Universidade Federal da Bahia, Salvador, Brazil

E-mail address: tertu@impa.br

Departamento de Matemática, Fac. Cs. Exactas y Naturales, UBA and imas-COniCET, Buenos Aires, Argentina

E-mail address: pgroisma@dm.uba.ar 